CRYSTALLOGRAPHIC COMMUNICATIONS

ISSN 2056-9890

Received 30 January 2018

Accepted 3 February 2018

Edited by D.-J. Xu, Zhejiang University (Yuquan Campus), China

Keywords: Schiff bas; crystal structure; DFT; 5-bromo-3-ethoxy-2-hydroxybenzaldehyde; 2-methoxyaniline.

CCDC reference: 1457124

Supporting information: this article has supporting information at journals.iucr.org/e

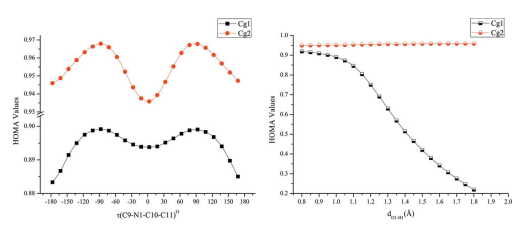

OPEN $\odot$ ACCESS

\section{Synthesis, crystal structure and computational studies of a new Schiff base compound: (E)-4-bromo-2-ethoxy-6-\{[(2-methoxyphenyl)- imino]methyl\}phenol}

\author{
Arzu Özek Yıldırım, ${ }^{a *}$ Murat Gülsu ${ }^{a}$ and Ciğdem Albayrak Kaștaș ${ }^{b}$
}

${ }^{\mathbf{a} D e p a r t m e n t}$ of Physics, Faculty of Arts and Sciences, Giresun University, Turkey, and ${ }^{\mathbf{b}}$ Department of Chemistry, Faculty of Arts and Sciences, Sinop University, Turkey. ${ }^{*}$ Correspondence e-mail: arzu.ozek.yildirim@giresun.edu.tr

The title compound, $\mathrm{C}_{16} \mathrm{H}_{16} \mathrm{BrNO}_{3}$, which shows enol-imine tautomerism, crystallizes in the monoclinic $P 2_{1} / c$ space group. All non- $\mathrm{H}$ atoms of the molecule are nearly coplanar, with a maximum deviation of 0.274 (3) $\AA$. In the crystal, molecules are held together by weak $\mathrm{C}-\mathrm{H} \cdots \mathrm{O}, \pi-\pi$ and $\mathrm{C}-\mathrm{H} \cdots \pi$ interactions. The $E / Z$ isomerism and enol/keto tautomerism energy barriers of the compound have been calculated by relaxed potential energy surface scan calculations with DFT methods. To observe the changes in the aromatic ring, HOMA aromaticity indexes were calculated during the scan process. Total energy and HOMA change curves were obtained to visualize results of the scan calculations.

\section{Chemical context}

The synthesis and chemistry of Schiff bases have received considerable attention over the last several decades, primarily owing to their remarkable potential pharmacological (Hu et al., 2012), anti-tumor (Kamel et al., 2010) and biological properties (Lozier et al., 1975). Furthermore, Schiff bases can display photo-chromic and thermo-chromic effect (Hadjoudis \& Mavridis, 2004). These effects depend on the prototropic tautomerism and molecular planarity in Schiff bases (Moustakali-Mavridis et al., 1978; Hadjoudis et al., 1987). Prototropic tautomerism emerges from the intramolecular $\mathrm{H}$-atom transfer between an enol-imine (Özdemir Tarı et al., 2016) and a keto-amine tautomer (Özek et al., 2006). The present work is part of our ongoing studies on Schiff bases (Özek Yıldırım et al., 2016, 2017; Albayrak et al., 2012). We report herein the synthesis, crystal structure and computational studies of the title compound, (E)-4-bromo-2-ethoxy-6-\{[(2-methoxyphenyl)imino]methyl phenol, obtained from the condensation of 5bromo-3-ethoxy-2-hydroxybenzaldehyde with 2-methoxyaniline.

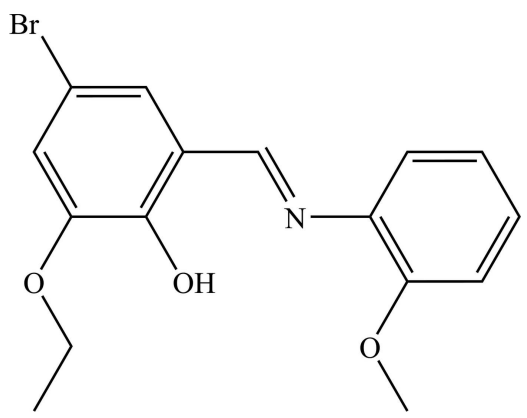




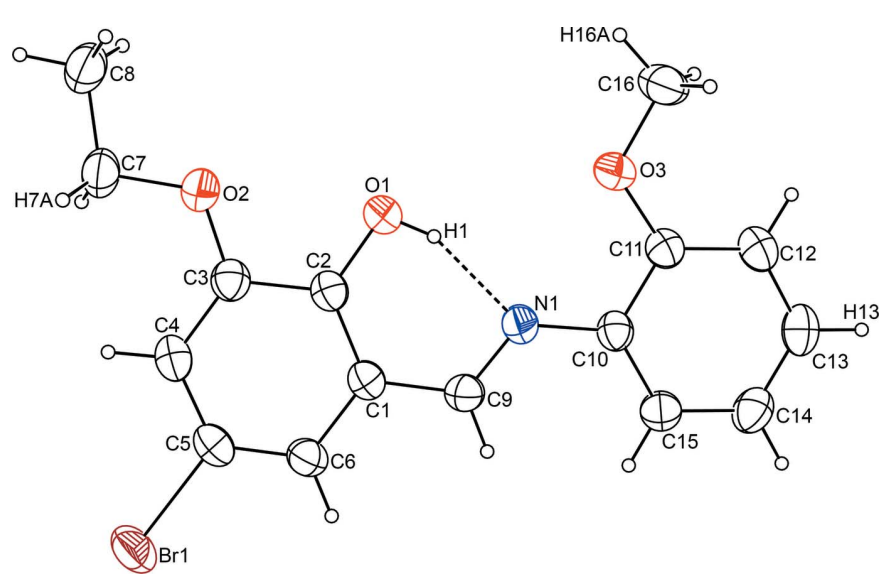

Figure 1

The molecular structure of the title compound, with atom labels and $50 \%$ probability displacement ellipsoids for non- $\mathrm{H}$ atoms. The dashed line indicates the intramolecular hydrogen bond.

\section{Structural commentary}

Fig. 1 represents the molecular structure of the title compound. All non-H atoms lie in the plane formed by the aromatic rings with a maximum deviation of 0.274 (3) $\AA$. The dihedral angle between the aromatic rings $\mathrm{C} 1-\mathrm{C} 6$ and $\mathrm{C} 10$ $\mathrm{C} 15$ is $2.25(13)^{\circ}$. In the chelate moiety, which comprises atoms $\mathrm{C} 1, \mathrm{C} 2, \mathrm{O} 1, \mathrm{H} 1, \mathrm{~N} 1$ and $\mathrm{C} 9, \mathrm{C} 9=\mathrm{N} 1[1.281(3)]$ is a typical double bond while $\mathrm{C} 2-\mathrm{O} 1[1.333$ (3)] is a typical single bond; these are similar to those in related structures (Petek et al., 2010; Gül et al., 2007). The harmonic oscillator model of aromaticity (HOMA; Kruszewski \& Krygowski, 1972) values were calculated $[0.88$ for $\mathrm{C} 1-\mathrm{C} 6$ and 0.98 for the $\mathrm{C} 10-\mathrm{C} 15$ ring] to observe the effect of substituent groups on the rings. There are no significant deformations of the rings when compared to those in (E)-2-ethoxy-6-[(2-methoxyphenyl-

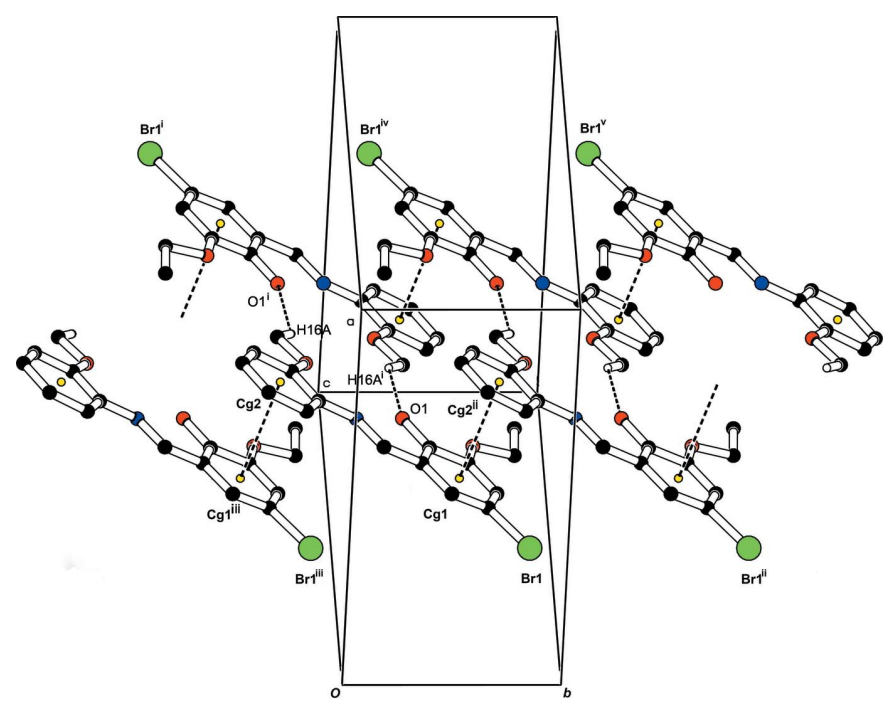

Figure 2

View of the inversion dimers, which are connected by $\pi-\pi$ interactions, propagating along the $c$-axis direction. [Symmetry codes: (i) $-x+1,-y$, $-z+1$; (ii) $x, y+1, z$; (iii) $x, y-1, z$; (iv) $-x+1,-y+1,-z+1$; (v) $-x+1$, $-y+2,-z+1$.]
Table 1

Hydrogen-bond geometry $\left(\AA,^{\circ}\right)$.

$C g 1$ and $C g 2$ are the centroids of the $\mathrm{C} 1-\mathrm{C} 6$ and $\mathrm{C} 10-\mathrm{C} 15$ rings, respectively.

\begin{tabular}{lllll}
\hline$D-\mathrm{H} \cdots A$ & $D-\mathrm{H}$ & $\mathrm{H} \cdots A$ & $D \cdots A$ & $D-\mathrm{H} \cdots A$ \\
\hline $\mathrm{O} 1-\mathrm{H} 1 \cdots \mathrm{N} 1$ & $0.81(5)$ & $1.80(5)$ & $2.566(3)$ & $157(5)$ \\
$\mathrm{C} 16-\mathrm{H} 16 A \cdots \mathrm{O} 1^{\mathrm{i}}$ & 0.96 & 2.55 & $3.293(3)$ & 135 \\
$\mathrm{C} 7-\mathrm{H} 7 A \cdots C g 1^{\text {ii }}$ & 0.97 & 2.80 & $3.662(3)$ & 149 \\
$\mathrm{C} 13-\mathrm{H} 13 \cdots C g 2^{\mathrm{iii}}$ & 0.93 & 2.79 & $3.629(3)$ & 150 \\
\hline
\end{tabular}

Symmetry codes:
$-x+\frac{3}{2}, y-\frac{1}{2},-z+\frac{1}{2}$.

imino)methyl]phenol (Petek et al., 2010). The chelate moiety forms an $S(6)$ graph-set motif through a strong intramolecular $\mathrm{O} 1-\mathrm{H} 1 \cdots \mathrm{N} 1$ hydrogen bond (Table 1$)$.

\section{Supramolecular features}

In the crystal, inversion dimers with an $R_{2}^{2}$ motif are generated by the weak $\mathrm{C} 16-\mathrm{H} 16 A \cdots \mathrm{O} 1(-x+1,-y,-z+1)$ hydrogen bonds (Table 1). As shown in Fig. 2, these dimers are connected to each other by $\pi-\pi$ interactions $[C g 1 \cdots C g 2(x$, $y+1, z)=3.6237$ (16) $\AA$; $C g 1$ and $C g 2$ are the centroids of the $\mathrm{C} 1-\mathrm{C} 6$ and $\mathrm{C} 10-\mathrm{C} 15$ rings, respectively]. $\mathrm{C}-\mathrm{H} \cdots \pi$ interactions (Table 1) generate zigzag chains along the [100] direction as shown in Fig. 3.

\section{Computational Studies}

Relaxed potential energy surface scan calculations were performed using the DFT/B3LYP/6-311G++(d,p) method with Gaussian 09W software (Frisch et al., 2009) to investigate the connection between the molecular conformation and physical properties of a Schiff base. The results of a torsional angle scan and a proton-transfer scan on the $\mathrm{O}-\mathrm{H} \cdots \mathrm{N}$ pathway are given in Fig. 4. The torsional barrier between the $E / Z$ isomers was found to be $1.94 \mathrm{kcal} \mathrm{mol}^{-1}$ and the enol-keto tauto-

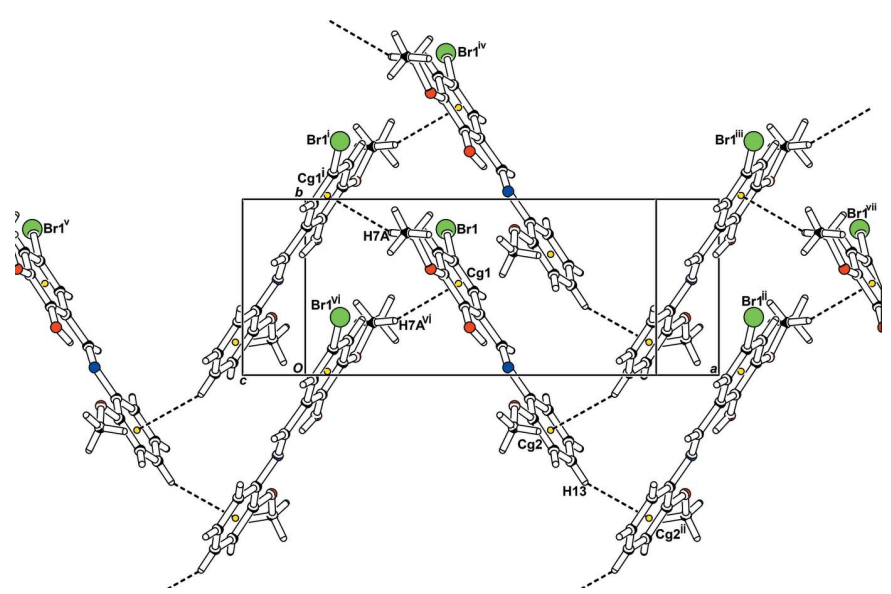

Figure 3

The packing, viewed down the $c$ axis, showing molecules connected by $\mathrm{C}-\mathrm{H} \cdots \pi$ interactions [Symmetry codes: (i) $-x+\frac{1}{2}, y+\frac{1}{2},-z+\frac{1}{2}$; (ii) $-x+\frac{3}{2}, y-\frac{1}{2},-z+\frac{1}{2}$; (iii) $-x+\frac{3}{2}, y+\frac{1}{2},-z+\frac{1}{2}$; (iv) $x, y+1, z$; (v) $x-1, y, z$; (vi) $-x+\frac{1}{2}, y-\frac{1}{2},-z+\frac{1}{2}$; (vii) $x+1, y, z$.] 

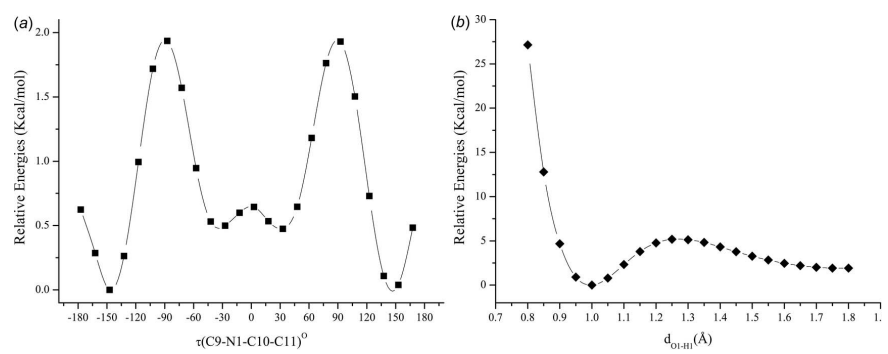

Figure 4

The potential energy curves for the torsional scan $(a)$ and the $\mathrm{O}-\mathrm{H}$ bond scan $(b)$. Relative energies are calculated with respect to the global minimum of each curve.

merism barrier was $1.92 \mathrm{kcal} \mathrm{mol}^{-1}$. The effects of the conformational changes on the aromatic ring can be visualized by calculating HOMA values during the scan calculations. Fig. $5 a$ shows that changes in the HOMA indices are very limited with an average fluctuation of $2 \%$. As can be seen in Fig. $5 b$, the aromaticity of the C1-C6 ring depends strongly on the prototropic tautomerism.

\section{Database survey}

A survey of the Cambridge Structural Database (CSD, Version 5.37, update May 2017; Groom et al., 2016) for the (E)-4-bromo-2-ethoxy-6-[(methylimino)methyl]phenol unit of the title compound reveals five compounds, viz. OCOVEK (Kaştaş et al., 2017a), OCOVIO (Kaştaş et al., 2017b), OCOVOU (Kaştaş et al., 2017c), OCOVUA (Kaştaş et al., 2017d) and LUWZIO (Özek Yıldırım et al., 2016). The molecular structures of the latter two compounds are planar, in which they are similar to the title compound, while the others are not planar.

\section{Synthesis and crystallization}

The title compound was prepared by refluxing a mixture of a solution containing 5-bromo-3-ethoxy-2-hydroxybenzaldehyde $(0.5 \mathrm{~g}, 2 \mathrm{mmol})$ in $20 \mathrm{ml}$ ethanol and a solution containing 2-methoxyaniline $(0.25 \mathrm{~g}, 2 \mathrm{mmol})$ in $20 \mathrm{ml}$ ethanol. The reaction mixture was stirred for $1 \mathrm{~h}$ under reflux. Crystals suitable for X-ray analysis were obtained from an ethanol solution by slow evaporation (yield 70\%).
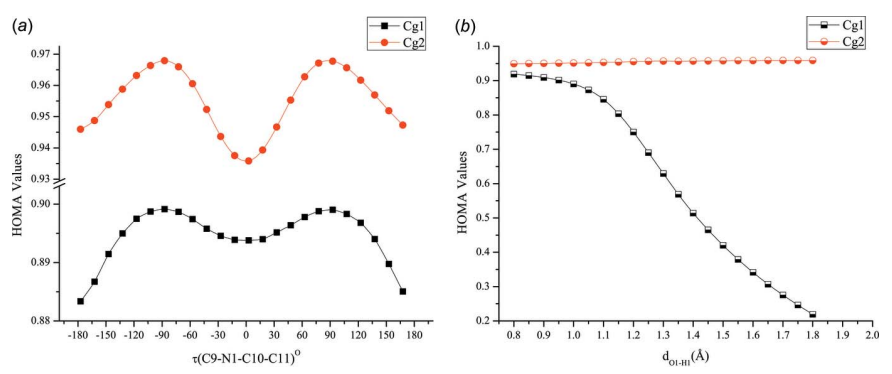

Figure 5

Graphics showing the variation of HOMA values with scan coordinate.
Table 2

Experimental details.

\begin{tabular}{|c|c|}
\hline \multicolumn{2}{|l|}{ Crystal data } \\
\hline Chemical formula & $\mathrm{C}_{16} \mathrm{H}_{16} \mathrm{BrNO}_{3}$ \\
\hline$M_{\mathrm{r}}$ & 350.21 \\
\hline Crystal system, space group & Monoclinic, $P 2_{1} / n$ \\
\hline Temperature $(\mathrm{K})$ & 296 \\
\hline$a, b, c(\AA)$ & $\begin{array}{l}15.3405(8), 6.5204(2), \\
\quad 15.3612(10)\end{array}$ \\
\hline$\beta\left(^{\circ}\right)$ & $98.716(5)$ \\
\hline$V\left(\AA^{3}\right)$ & $1518.78(14)$ \\
\hline$Z$ & 4 \\
\hline Radiation type & Мо $K \alpha$ \\
\hline$\mu\left(\mathrm{mm}^{-1}\right)$ & 2.72 \\
\hline Crystal size (mm) & $0.56 \times 0.28 \times 0.05$ \\
\hline \multicolumn{2}{|l|}{ Data collection } \\
\hline Diffractometer & Stoe IPDS 2 \\
\hline Absorption correction & $\begin{array}{l}\text { Integration ( } X \text {-RED32; Stoe \& Cie, } \\
\text { 2002) }\end{array}$ \\
\hline$T_{\min }, T_{\max }$ & $0.437,0.893$ \\
\hline $\begin{array}{l}\text { No. of measured, independent and } \\
\text { observed }[I>2 \sigma(I)] \text { reflections }\end{array}$ & $18156,3491,2754$ \\
\hline$R_{\text {int }}$ & 0.042 \\
\hline$(\sin \theta / \lambda)_{\max }\left(\AA^{-1}\right)$ & 0.650 \\
\hline \multicolumn{2}{|l|}{ Refinement } \\
\hline$R\left[F^{2}>2 \sigma\left(F^{2}\right)\right], w R\left(F^{2}\right), S$ & $0.042,0.091,1.06$ \\
\hline No. of reflections & 3491 \\
\hline No. of parameters & 194 \\
\hline $\mathrm{H}$-atom treatment & $\begin{array}{l}\mathrm{H} \text { atoms treated by a mixture of } \\
\text { independent and constrained } \\
\text { refinement }\end{array}$ \\
\hline$\Delta \rho_{\max }, \Delta \rho_{\min }\left({\left.\mathrm{e} \AA^{-3}\right)}^{-3}\right.$ & $0.28,-0.38$ \\
\hline
\end{tabular}

Computer programs: $X$-AREA and X-RED32 (Stoe \& Cie, 2002), SHELXS97 (Sheldrick, 2015), SHELXL2018 (Sheldrick, 2015), ORTEP-3 for Windows and WinGX (Farrugia, 2012) and PLATON (Spek, 2009).

\section{Refinement}

Crystal data, data collection and structure refinement details are summarized in Table 2. The hydroxyl atom $\mathrm{H} 1$ was refined freely. All the other $\mathrm{H}$ atoms were located geometrically and refined using a riding model with $\mathrm{C}-\mathrm{H}=0.93-0.97 \AA U_{\text {iso }}(\mathrm{H})$ $=1.2 U_{\text {eq }}(\mathrm{C})$.

\section{Acknowledgements}

The authors thank Professor Orhan Büyükgüngör for his guidance in this study.

\section{Funding information}

Funding for this research was provided by: Giresun University (FEN-BAP-A-250414-75).

\section{References}

Albayrak, Ç., Odabaşoğlu, M., Özek, A. \& Büyükgüngör, O. (2012). Spectrochim. Acta A, 85, 85-91.

Farrugia, L. J. (2012). J. Appl. Cryst. 45, 849-854.

Frisch, M. J., Trucks, G. W., Schlegel, H. B., Scuseria, G. E., Robb, M. A., Cheeseman, J. R., Scalmani, G., Barone, V., Mennucci, B., Petersson, G. A., Nakatsuji, H., Caricato, M., Li, X., Hratchian, H. P., Izmaylov, A. F., Bloino, J., Zheng, G., Sonnenberg, J. L., Hada, M., Ehara, M., Toyota, K., Fukuda, R., Hasegawa, J., Ishida, M., Nakajima, T., Honda, Y., Kitao, O., Nakai, H., Vreven, T., Montgomery, J. A. Jr, Peralta, J. E., Ogliaro, F., Bearpark, M., 
Heyd, J. J., Brothers, E., Kudin, K. N., Staroverov, V. N., Keith, T., Kobayashi, R., Normand, J., Raghavachari, K., Rendell, A., Burant, J. C., Iyengar, S. S., Tomasi, J., Cossi, M., Rega, N., Millam, J. M., Klene, M., Knox, J. E., Cross, J. B., Bakken, V., Adamo, C., Jaramillo, J., Gomperts, R., Stratmann, R. E., Yazyev, O., Austin, A. J., Cammi, R., Pomelli, C., Ochterski, J. W., Martin, R. L., Morokuma, K., Zakrzewski, V. G., Voth, G. A., Salvador, P., Dannenberg, J. J., Dapprich, S., Daniels, A. D., Farkas, O., Foresman, J. B., Ortiz, J. V., Cioslowski, J. \& Fox, D. J. (2009). GAUSSIAN09. Gaussian Inc., USA.

Groom, C. R., Bruno, I. J., Lightfoot, M. P. \& Ward, S. C. (2016). Acta Cryst. B72, 171-179.

Gül, Z. S., Ağar, A. A. \& Işık, Ş. (2007). Acta Cryst. E63, o4564.

Hadjoudis, E. \& Mavridis, I. M. (2004). Chem. Soc. Rev. 33, 579-588.

Hadjoudis, E., Vittorakis, M. \& Moustakali-Mavridis, I. (1987). Tetrahedron, 43, 1345-1360.

Hu, G., Wang, G., Duan, N., Wen, X., Cao, T., Xie, S. \& Huang, W. (2012). Acta Pharmaceutica Sinica B, 2(3), 312-317.

Kamel, M. M., Ali, H. I., Anwar, M. M., Mohamed, N. A. \& Soliman, A. M. (2010). Eur. J. Med. Chem. 45, 572-580.

Kaştaş, G., Albayrak Kaştaş, Ç. \& Frank, R. (2017a). CSD Communication, https://doi. org/10.5517/ccdc.csd.cc12bq15.

Kaştaş, G., Albayrak Kaştaş, Ç. \& Frank, R. (2017b). CSD Communication, https://doi. org/10.5517/ccdc.csd.cc12bq37.
Kaştaş, G., Albayrak Kaştaş, Ç. \& Frank, R. (2017c). CSD Communication, https://doi. org/10.5517/ccdc.csd.cc12bq59.

Kaştaş, G., Albayrak Kaştaş, Ç. \& Frank, R. (2017d). CSD Communication, https://doi. org/10.5517/ccdc.csd.cc12bq6b.

Kruszewski, J. \& Krygowski, T. M. (1972). Tetrahedron Lett. 13, 38393842.

Lozier, R. H., Bogomolni, R. A. \& Stoeckenius, W. (1975). Biophys. J. 15, 955-962.

Moustakali-Mavridis, I., Hadjoudis, E. \& Mavridis, A. (1978). Acta Cryst. B34, 3709-3715.

Özdemir Tarı, G., Ceylan, Ü., Ümit, , Ağar, E. \& Eserci, H. (2016). J. Mol. Struct. 1126, 83-93.

Özek, A., Albayrak, C., Odabaşoğlu, M. \& Büyükgüngör, O. (2006). Acta Cryst. C62, o173-o177.

Özek Yıldırım, A., Albayrak Kaştaş, Ç. \& Gülsu, M. (2016). J. Mol. Struct. 1103, 311-318.

Özek Yıldırım, A., Yıldırım, M. H. \& Albayrak Kaştaş, Ç. (2017). J. Mol. Struct. 1127, 275-282.

Petek, H., Albayrak, Ç., Odabaşoğlu, M., Şenel, İ. \& Büyükgüngör, O. (2010). Struct. Chem. 21, 681-690.

Sheldrick, G. M. (2015). Acta Cryst. C71, 3-8.

Spek, A. L. (2009). Acta Cryst. D65, 148-155.

Stoe \& Cie (2002). $X$-AREA and $X$-RED32. Stoe \& Cie, Germany. 


\section{supporting information}

Acta Cryst. (2018). E74, 319-322 [https://doi.org/10.1107/S2056989018002062]

Synthesis, crystal structure and computational studies of a new Schiff base compound: $(E)-4-b r o m o-2-e t h o x y-6-\{[(2-m e t h o x y p h e n y l) i m i n o] m e t h y l\}$ phenol

\section{Arzu Özek Yıldırım, Murat Gülsu and Çiğdem Albayrak Kaştaş}

Computing details

Data collection: $X$-AREA (Stoe \& Cie, 2002); cell refinement: $X$-AREA (Stoe \& Cie, 2002); data reduction: $X$-RED32

(Stoe \& Cie, 2002); program(s) used to solve structure: SHELXS97 (Sheldrick, 2015); program(s) used to refine structure: SHELXL2018 (Sheldrick, 2015); molecular graphics: ORTEP-3 for Windows (Farrugia, 2012) and PLATON (Spek, 2009); software used to prepare material for publication: $\operatorname{Win} G X$ (Farrugia, 2012).

(E)-4-Bromo-2-ethoxy-6-\{[(2-methoxyphenyl)imino]methyl\}phenol

Crystal data

$\mathrm{C}_{16} \mathrm{H}_{16} \mathrm{BrNO}_{3}$

$M_{r}=350.21$

Monoclinic, $P 2{ }_{1} / n$

$a=15.3405(8) \AA$

$b=6.5204(2) \AA$

$c=15.3612(10) \AA$

$\beta=98.716(5)^{\circ}$

$V=1518.78(14) \AA^{3}$

$Z=4$

Data collection

Stoe IPDS 2 diffractometer

Radiation source: sealed X-ray tube, $12 \times 0.4$ $\mathrm{mm}$ long-fine focus

Detector resolution: 6.67 pixels $\mathrm{mm}^{-1}$ rotation method scans

Absorption correction: integration

(X-RED32; Stoe \& Cie, 2002)

$T_{\min }=0.437, T_{\max }=0.893$

Refinement

Refinement on $F^{2}$

Least-squares matrix: full

$R\left[F^{2}>2 \sigma\left(F^{2}\right)\right]=0.042$

$w R\left(F^{2}\right)=0.091$

$S=1.06$

3491 reflections

194 parameters

0 restraints
$F(000)=712$

$D_{\mathrm{x}}=1.532 \mathrm{Mg} \mathrm{m}^{-3}$

Mo $K \alpha$ radiation, $\lambda=0.71073 \AA$

Cell parameters from 3491 reflections

$\theta=2.0-28.1^{\circ}$

$\mu=2.72 \mathrm{~mm}^{-1}$

$T=296 \mathrm{~K}$

Prism, orange

$0.56 \times 0.28 \times 0.05 \mathrm{~mm}$

18156 measured reflections

3491 independent reflections

2754 reflections with $I>2 \sigma(I)$

$R_{\text {int }}=0.042$

$\theta_{\max }=27.5^{\circ}, \theta_{\min }=2.0^{\circ}$

$h=-19 \rightarrow 19$

$k=-8 \rightarrow 8$

$l=-19 \rightarrow 19$

Hydrogen site location: mixed

$\mathrm{H}$ atoms treated by a mixture of independent and constrained refinement

$w=1 /\left[\sigma^{2}\left(F_{\mathrm{o}}^{2}\right)+(0.0398 P)^{2}+0.5265 P\right]$

where $P=\left(F_{\mathrm{o}}{ }^{2}+2 F_{\mathrm{c}}{ }^{2}\right) / 3$

$(\Delta / \sigma)_{\max }=0.001$

$\Delta \rho_{\max }=0.28$ e $\AA^{-3}$

$\Delta \rho_{\min }=-0.38$ e $\AA^{-3}$ 


\section{Special details}

Geometry. All esds (except the esd in the dihedral angle between two 1.s. planes) are estimated using the full covariance matrix. The cell esds are taken into account individually in the estimation of esds in distances, angles and torsion angles; correlations between esds in cell parameters are only used when they are defined by crystal symmetry. An approximate (isotropic) treatment of cell esds is used for estimating esds involving l.s. planes.

Fractional atomic coordinates and isotropic or equivalent isotropic displacement parameters $\left(\hat{A}^{2}\right)$

\begin{tabular}{|c|c|c|c|c|}
\hline & $x$ & $y$ & $z$ & $U_{\text {iso }} * / U_{\text {eq }}$ \\
\hline $\mathrm{C} 1$ & $0.44857(16)$ & $0.3446(4)$ & $0.18253(15)$ & $0.0403(5)$ \\
\hline $\mathrm{C} 2$ & $0.42497(17)$ & 0.3902 (4) & $0.26511(16)$ & $0.0417(5)$ \\
\hline $\mathrm{C} 3$ & $0.37588(17)$ & $0.5714(4)$ & $0.27491(17)$ & $0.0440(6)$ \\
\hline $\mathrm{C} 4$ & $0.35124(17)$ & $0.6978(4)$ & $0.20324(18)$ & $0.0469(6)$ \\
\hline H4 & 0.318414 & 0.815670 & 0.208919 & $0.056^{*}$ \\
\hline $\mathrm{C} 5$ & $0.37596(17)$ & $0.6473(4)$ & $0.12266(17)$ & $0.0456(6)$ \\
\hline C6 & $0.42285(18)$ & $0.4758(4)$ & $0.11072(16)$ & $0.0457(6)$ \\
\hline H6 & 0.437777 & 0.445619 & 0.055674 & $0.055^{*}$ \\
\hline $\mathrm{C} 7$ & $0.3190(2)$ & $0.7984(4)$ & $0.3743(2)$ & $0.0574(7)$ \\
\hline H7A & 0.260045 & 0.809407 & 0.341291 & $0.069^{*}$ \\
\hline H7B & 0.354488 & 0.909669 & 0.356734 & $0.069^{*}$ \\
\hline $\mathrm{C} 8$ & $0.3155(2)$ & $0.8097(5)$ & $0.4713(2)$ & $0.0640(8)$ \\
\hline H8A & 0.290087 & 0.938248 & 0.484727 & $0.077^{*}$ \\
\hline H8B & 0.280127 & 0.699194 & 0.487896 & $0.077^{*}$ \\
\hline $\mathrm{H} 8 \mathrm{C}$ & 0.374171 & 0.799032 & 0.503274 & $0.077^{*}$ \\
\hline C9 & $0.50092(17)$ & $0.1655(4)$ & $0.17069(16)$ & $0.0442(5)$ \\
\hline H9 & 0.516428 & 0.138217 & 0.115620 & $0.053^{*}$ \\
\hline $\mathrm{C} 10$ & $0.57777(16)$ & $-0.1337(4)$ & $0.22892(16)$ & $0.0406(5)$ \\
\hline $\mathrm{C} 11$ & $0.59676(16)$ & $-0.2499(4)$ & $0.30608(16)$ & $0.0420(5)$ \\
\hline $\mathrm{C} 12$ & 0.64681 (19) & $-0.4279(4)$ & $0.30608(19)$ & $0.0513(6)$ \\
\hline H12 & 0.659509 & -0.505656 & 0.357194 & $0.062 *$ \\
\hline $\mathrm{C} 13$ & $0.67759(18)$ & $-0.4892(5)$ & $0.2304(2)$ & $0.0550(7)$ \\
\hline H13 & 0.711082 & -0.608217 & 0.230857 & $0.066^{*}$ \\
\hline $\mathrm{C} 14$ & $0.6594(2)$ & $-0.3766(5)$ & $0.1542(2)$ & $0.0552(7)$ \\
\hline H14 & 0.680358 & -0.419005 & 0.103432 & $0.066^{*}$ \\
\hline $\mathrm{C} 15$ & 0.60944 (19) & $-0.1994(4)$ & $0.15371(18)$ & $0.0508(6)$ \\
\hline H15 & 0.596978 & -0.123232 & 0.102130 & $0.061^{*}$ \\
\hline $\mathrm{C} 16$ & $0.5727(2)$ & $-0.2971(5)$ & $0.45460(19)$ & $0.0622(8)$ \\
\hline H16A & 0.546330 & -0.227372 & 0.499090 & $0.075^{*}$ \\
\hline H16B & 0.544000 & -0.426840 & 0.441808 & $0.075^{*}$ \\
\hline $\mathrm{H} 16 \mathrm{C}$ & 0.634215 & -0.319190 & 0.475376 & $0.075^{*}$ \\
\hline N1 & $0.52630(14)$ & $0.0438(3)$ & $0.23498(13)$ & $0.0423(5)$ \\
\hline $\mathrm{O} 1$ & $0.44838(15)$ & $0.2719(3)$ & $0.33548(12)$ & $0.0536(5)$ \\
\hline $\mathrm{O} 2$ & $0.35740(14)$ & $0.6041(3)$ & $0.35724(12)$ & $0.0546(5)$ \\
\hline $\mathrm{O} 3$ & $0.56318(14)$ & $-0.1756(3)$ & $0.37679(12)$ & $0.0558(5)$ \\
\hline Br1 & $0.34398(2)$ & $0.82939(5)$ & $0.02613(2)$ & $0.06603(13)$ \\
\hline H1 & $0.479(3)$ & $0.186(8)$ & $0.316(3)$ & $0.119(18)^{*}$ \\
\hline
\end{tabular}


Atomic displacement parameters $\left(\AA^{2}\right)$

\begin{tabular}{lllllll}
\hline & $U^{11}$ & $U^{22}$ & $U^{33}$ & $U^{12}$ & $U^{13}$ & $U^{23}$ \\
\hline C1 & $0.0405(12)$ & $0.0374(12)$ & $0.0424(12)$ & $-0.0003(11)$ & $0.0041(10)$ & $0.0031(10)$ \\
C2 & $0.0433(13)$ & $0.0374(13)$ & $0.0443(12)$ & $0.0007(10)$ & $0.0064(10)$ & $0.0027(10)$ \\
C3 & $0.0429(14)$ & $0.0415(13)$ & $0.0483(13)$ & $0.0002(11)$ & $0.0092(11)$ & $0.0009(11)$ \\
C4 & $0.0441(14)$ & $0.0383(14)$ & $0.0583(15)$ & $0.0045(11)$ & $0.0081(11)$ & $0.0060(11)$ \\
C5 & $0.0446(14)$ & $0.0415(14)$ & $0.0493(13)$ & $-0.0018(11)$ & $0.0021(11)$ & $0.0109(11)$ \\
C6 & $0.0492(14)$ & $0.0476(15)$ & $0.0403(12)$ & $0.0025(12)$ & $0.0070(11)$ & $0.0067(11)$ \\
C7 & $0.0640(18)$ & $0.0433(16)$ & $0.0674(18)$ & $0.0125(13)$ & $0.0184(14)$ & $0.0005(13)$ \\
C8 & $0.074(2)$ & $0.0519(17)$ & $0.0698(19)$ & $0.0103(15)$ & $0.0230(16)$ & $-0.0074(15)$ \\
C9 & $0.0514(14)$ & $0.0430(13)$ & $0.0387(12)$ & $-0.0004(12)$ & $0.0087(10)$ & $0.0010(11)$ \\
C10 & $0.0404(13)$ & $0.0363(13)$ & $0.0458(12)$ & $-0.0001(10)$ & $0.0089(10)$ & $0.0016(10)$ \\
C11 & $0.0392(13)$ & $0.0422(13)$ & $0.0450(12)$ & $-0.0001(11)$ & $0.0080(10)$ & $0.0031(10)$ \\
C12 & $0.0491(15)$ & $0.0465(15)$ & $0.0576(15)$ & $0.0062(12)$ & $0.0061(12)$ & $0.0089(13)$ \\
C13 & $0.0458(15)$ & $0.0433(15)$ & $0.0764(19)$ & $0.0074(12)$ & $0.0106(14)$ & $-0.0017(14)$ \\
C14 & $0.0555(17)$ & $0.0534(17)$ & $0.0607(16)$ & $0.0041(13)$ & $0.0217(13)$ & $-0.0073(13)$ \\
C15 & $0.0580(16)$ & $0.0498(16)$ & $0.0475(14)$ & $0.0037(13)$ & $0.0170(12)$ & $0.0037(12)$ \\
C16 & $0.070(2)$ & $0.070(2)$ & $0.0473(15)$ & $0.0027(16)$ & $0.0107(13)$ & $0.0152(14)$ \\
N1 & $0.0467(12)$ & $0.0375(11)$ & $0.0436(10)$ & $0.0035(9)$ & $0.0092(9)$ & $0.0030(9)$ \\
O1 & $0.0730(14)$ & $0.0473(11)$ & $0.0425(10)$ & $0.0160(10)$ & $0.0152(9)$ & $0.0081(8)$ \\
O2 & $0.0703(13)$ & $0.0445(10)$ & $0.0519(10)$ & $0.0137(9)$ & $0.0184(9)$ & $0.0025(8)$ \\
O3 & $0.0709(13)$ & $0.0559(11)$ & $0.0434(9)$ & $0.0145(10)$ & $0.0176(9)$ & $0.0106(9)$ \\
B1 & $0.0763(2)$ & $0.05858(19)$ & $0.06202(19)$ & $0.01265(16)$ & $0.00668(14)$ & $0.02361(15)$ \\
& & & & & &
\end{tabular}

Geometric parameters $\left(\AA,{ }^{\circ}\right)$

\begin{tabular}{llll}
\hline $\mathrm{C} 1-\mathrm{C} 2$ & $1.403(3)$ & $\mathrm{C} 9-\mathrm{N} 1$ & $1.281(3)$ \\
$\mathrm{C} 1-\mathrm{C} 6$ & $1.405(3)$ & $\mathrm{C} 9-\mathrm{H} 9$ & 0.9300 \\
$\mathrm{C} 1-\mathrm{C} 9$ & $1.444(4)$ & $\mathrm{C} 10-\mathrm{C} 15$ & $1.387(4)$ \\
$\mathrm{C} 2-\mathrm{O} 1$ & $1.333(3)$ & $\mathrm{C} 10-\mathrm{C} 11$ & $1.400(3)$ \\
$\mathrm{C} 2-\mathrm{C} 3$ & $1.421(4)$ & $\mathrm{C} 10-\mathrm{N} 1$ & $1.412(3)$ \\
$\mathrm{C} 3-\mathrm{O} 2$ & $1.354(3)$ & $\mathrm{C} 11-\mathrm{O} 3$ & $1.360(3)$ \\
$\mathrm{C} 3-\mathrm{C} 4$ & $1.381(4)$ & $\mathrm{C} 11-\mathrm{C} 12$ & $1.392(4)$ \\
$\mathrm{C} 4-\mathrm{C} 5$ & $1.388(4)$ & $\mathrm{C} 12-\mathrm{C} 13$ & $1.379(4)$ \\
$\mathrm{C} 4-\mathrm{H} 4$ & 0.9300 & $\mathrm{C} 12-\mathrm{H} 12$ & 0.9300 \\
$\mathrm{C} 5-\mathrm{C} 6$ & $1.356(4)$ & $\mathrm{C} 13-\mathrm{C} 14$ & $1.373(4)$ \\
$\mathrm{C} 5-\mathrm{Br} 1$ & $1.905(2)$ & $\mathrm{C} 13-\mathrm{H} 13$ & 0.9300 \\
$\mathrm{C} 6-\mathrm{H} 6$ & 0.9300 & $\mathrm{C} 14-\mathrm{C} 15$ & $1.386(4)$ \\
$\mathrm{C} 7-\mathrm{O} 2$ & $1.438(3)$ & $\mathrm{C} 14-\mathrm{H} 14$ & 0.9300 \\
$\mathrm{C} 7-\mathrm{C} 8$ & $1.500(4)$ & $\mathrm{C} 15-\mathrm{H} 15$ & 1.9300 \\
$\mathrm{C} 7-\mathrm{H} 7 \mathrm{~A}$ & 0.9700 & $\mathrm{C} 16-\mathrm{O} 3$ & 0.9600 \\
$\mathrm{C} 7-\mathrm{H} 7 \mathrm{~B}$ & 0.9700 & $\mathrm{C} 16-\mathrm{H} 16 \mathrm{~A}$ & 0.9600 \\
$\mathrm{C} 8-\mathrm{H} 8 \mathrm{~A}$ & 0.9600 & $\mathrm{C} 16-\mathrm{H} 16 \mathrm{~B}$ & 0.9600 \\
$\mathrm{C} 8-\mathrm{H} 8 \mathrm{~B}$ & 0.9600 & $\mathrm{C} 16-\mathrm{H} 16 \mathrm{C}$ & $0.81(5)$ \\
$\mathrm{C} 8-\mathrm{H} 8 \mathrm{C}$ & 0.9600 & $\mathrm{O} 1-\mathrm{H} 1$ & 119.5 \\
$\mathrm{C} 2-\mathrm{C} 1-\mathrm{C} 6$ & & &
\end{tabular}




\begin{tabular}{|c|c|c|c|}
\hline $\mathrm{C} 2-\mathrm{C} 1-\mathrm{C} 9$ & $120.7(2)$ & $\mathrm{C} 1-\mathrm{C} 9-\mathrm{H} 9$ & 119.5 \\
\hline $\mathrm{C} 6-\mathrm{C} 1-\mathrm{C} 9$ & $119.3(2)$ & $\mathrm{C} 15-\mathrm{C} 10-\mathrm{C} 11$ & $118.9(2)$ \\
\hline $\mathrm{O} 1-\mathrm{C} 2-\mathrm{C} 1$ & $122.4(2)$ & $\mathrm{C} 15-\mathrm{C} 10-\mathrm{N} 1$ & $125.3(2)$ \\
\hline $\mathrm{O} 1-\mathrm{C} 2-\mathrm{C} 3$ & $118.5(2)$ & $\mathrm{C} 11-\mathrm{C} 10-\mathrm{N} 1$ & $115.8(2)$ \\
\hline $\mathrm{C} 1-\mathrm{C} 2-\mathrm{C} 3$ & $119.1(2)$ & $\mathrm{O} 3-\mathrm{C} 11-\mathrm{C} 12$ & $125.0(2)$ \\
\hline $\mathrm{O} 2-\mathrm{C} 3-\mathrm{C} 4$ & $125.4(2)$ & $\mathrm{O} 3-\mathrm{C} 11-\mathrm{C} 10$ & $115.3(2)$ \\
\hline $\mathrm{O} 2-\mathrm{C} 3-\mathrm{C} 2$ & $114.8(2)$ & $\mathrm{C} 12-\mathrm{C} 11-\mathrm{C} 10$ & $119.7(2)$ \\
\hline $\mathrm{C} 4-\mathrm{C} 3-\mathrm{C} 2$ & $119.8(2)$ & $\mathrm{C} 13-\mathrm{C} 12-\mathrm{C} 11$ & $120.1(3)$ \\
\hline $\mathrm{C} 3-\mathrm{C} 4-\mathrm{C} 5$ & $119.4(2)$ & $\mathrm{C} 13-\mathrm{C} 12-\mathrm{H} 12$ & 120.0 \\
\hline $\mathrm{C} 3-\mathrm{C} 4-\mathrm{H} 4$ & 120.3 & $\mathrm{C} 11-\mathrm{C} 12-\mathrm{H} 12$ & 120.0 \\
\hline $\mathrm{C} 5-\mathrm{C} 4-\mathrm{H} 4$ & 120.3 & $\mathrm{C} 14-\mathrm{C} 13-\mathrm{C} 12$ & $120.8(3)$ \\
\hline $\mathrm{C} 6-\mathrm{C} 5-\mathrm{C} 4$ & $122.6(2)$ & $\mathrm{C} 14-\mathrm{C} 13-\mathrm{H} 13$ & 119.6 \\
\hline $\mathrm{C} 6-\mathrm{C} 5-\mathrm{Br} 1$ & $119.2(2)$ & $\mathrm{C} 12-\mathrm{C} 13-\mathrm{H} 13$ & 119.6 \\
\hline $\mathrm{C} 4-\mathrm{C} 5-\mathrm{Br} 1$ & $118.23(19)$ & $\mathrm{C} 13-\mathrm{C} 14-\mathrm{C} 15$ & $119.5(3)$ \\
\hline $\mathrm{C} 5-\mathrm{C} 6-\mathrm{C} 1$ & $119.2(2)$ & $\mathrm{C} 13-\mathrm{C} 14-\mathrm{H} 14$ & 120.3 \\
\hline $\mathrm{C} 5-\mathrm{C} 6-\mathrm{H} 6$ & 120.4 & $\mathrm{C} 15-\mathrm{C} 14-\mathrm{H} 14$ & 120.3 \\
\hline $\mathrm{C} 1-\mathrm{C} 6-\mathrm{H} 6$ & 120.4 & $\mathrm{C} 14-\mathrm{C} 15-\mathrm{C} 10$ & $121.1(3)$ \\
\hline $\mathrm{O} 2-\mathrm{C} 7-\mathrm{C} 8$ & $107.6(2)$ & $\mathrm{C} 14-\mathrm{C} 15-\mathrm{H} 15$ & 119.5 \\
\hline $\mathrm{O} 2-\mathrm{C} 7-\mathrm{H} 7 \mathrm{~A}$ & 110.2 & $\mathrm{C} 10-\mathrm{C} 15-\mathrm{H} 15$ & 119.5 \\
\hline $\mathrm{C} 8-\mathrm{C} 7-\mathrm{H} 7 \mathrm{~A}$ & 110.2 & $\mathrm{O} 3-\mathrm{C} 16-\mathrm{H} 16 \mathrm{~A}$ & 109.5 \\
\hline $\mathrm{O} 2-\mathrm{C} 7-\mathrm{H} 7 \mathrm{~B}$ & 110.2 & $\mathrm{O} 3-\mathrm{C} 16-\mathrm{H} 16 \mathrm{~B}$ & 109.5 \\
\hline $\mathrm{C} 8-\mathrm{C} 7-\mathrm{H} 7 \mathrm{~B}$ & 110.2 & $\mathrm{H} 16 \mathrm{~A}-\mathrm{C} 16-\mathrm{H} 16 \mathrm{~B}$ & 109.5 \\
\hline $\mathrm{H} 7 \mathrm{~A}-\mathrm{C} 7-\mathrm{H} 7 \mathrm{~B}$ & 108.5 & $\mathrm{O} 3-\mathrm{C} 16-\mathrm{H} 16 \mathrm{C}$ & 109.5 \\
\hline $\mathrm{C} 7-\mathrm{C} 8-\mathrm{H} 8 \mathrm{~A}$ & 109.5 & $\mathrm{H} 16 \mathrm{~A}-\mathrm{C} 16-\mathrm{H} 16 \mathrm{C}$ & 109.5 \\
\hline $\mathrm{C} 7-\mathrm{C} 8-\mathrm{H} 8 \mathrm{~B}$ & 109.5 & $\mathrm{H} 16 \mathrm{~B}-\mathrm{C} 16-\mathrm{H} 16 \mathrm{C}$ & 109.5 \\
\hline $\mathrm{H} 8 \mathrm{~A}-\mathrm{C} 8-\mathrm{H} 8 \mathrm{~B}$ & 109.5 & $\mathrm{C} 9-\mathrm{N} 1-\mathrm{C} 10$ & $124.4(2)$ \\
\hline $\mathrm{C} 7-\mathrm{C} 8-\mathrm{H} 8 \mathrm{C}$ & 109.5 & $\mathrm{C} 2-\mathrm{O} 1-\mathrm{H} 1$ & $102(3)$ \\
\hline $\mathrm{H} 8 \mathrm{~A}-\mathrm{C} 8-\mathrm{H} 8 \mathrm{C}$ & 109.5 & $\mathrm{C} 3-\mathrm{O} 2-\mathrm{C} 7$ & $117.3(2)$ \\
\hline $\mathrm{H} 8 \mathrm{~B}-\mathrm{C} 8-\mathrm{H} 8 \mathrm{C}$ & 109.5 & $\mathrm{C} 11-\mathrm{O} 3-\mathrm{C} 16$ & $118.0(2)$ \\
\hline $\mathrm{N} 1-\mathrm{C} 9-\mathrm{C} 1$ & $120.9(2)$ & & \\
\hline $\mathrm{C} 6-\mathrm{C} 1-\mathrm{C} 2-\mathrm{O} 1$ & $-179.4(2)$ & $\mathrm{N} 1-\mathrm{C} 10-\mathrm{C} 11-\mathrm{O} 3$ & $-0.2(3)$ \\
\hline $\mathrm{C} 9-\mathrm{C} 1-\mathrm{C} 2-\mathrm{O} 1$ & $-0.7(4)$ & $\mathrm{C} 15-\mathrm{C} 10-\mathrm{C} 11-\mathrm{C} 12$ & $0.1(4)$ \\
\hline $\mathrm{C} 6-\mathrm{C} 1-\mathrm{C} 2-\mathrm{C} 3$ & $-0.7(4)$ & $\mathrm{N} 1-\mathrm{C} 10-\mathrm{C} 11-\mathrm{C} 12$ & $179.7(2)$ \\
\hline $\mathrm{C} 9-\mathrm{C} 1-\mathrm{C} 2-\mathrm{C} 3$ & $177.9(2)$ & $\mathrm{O} 3-\mathrm{C} 11-\mathrm{C} 12-\mathrm{C} 13$ & $180.0(3)$ \\
\hline $\mathrm{O} 1-\mathrm{C} 2-\mathrm{C} 3-\mathrm{O} 2$ & $-0.3(4)$ & $\mathrm{C} 10-\mathrm{C} 11-\mathrm{C} 12-\mathrm{C} 13$ & $0.1(4)$ \\
\hline $\mathrm{C} 1-\mathrm{C} 2-\mathrm{C} 3-\mathrm{O} 2$ & $-179.0(2)$ & $\mathrm{C} 11-\mathrm{C} 12-\mathrm{C} 13-\mathrm{C} 14$ & $-0.1(4)$ \\
\hline $\mathrm{O} 1-\mathrm{C} 2-\mathrm{C} 3-\mathrm{C} 4$ & $179.5(2)$ & $\mathrm{C} 12-\mathrm{C} 13-\mathrm{C} 14-\mathrm{C} 15$ & $0.0(5)$ \\
\hline $\mathrm{C} 1-\mathrm{C} 2-\mathrm{C} 3-\mathrm{C} 4$ & $0.8(4)$ & $\mathrm{C} 13-\mathrm{C} 14-\mathrm{C} 15-\mathrm{C} 10$ & $0.1(5)$ \\
\hline $\mathrm{O} 2-\mathrm{C} 3-\mathrm{C} 4-\mathrm{C} 5$ & $179.0(2)$ & $\mathrm{C} 11-\mathrm{C} 10-\mathrm{C} 15-\mathrm{C} 14$ & $-0.2(4)$ \\
\hline $\mathrm{C} 2-\mathrm{C} 3-\mathrm{C} 4-\mathrm{C} 5$ & $-0.9(4)$ & $\mathrm{N} 1-\mathrm{C} 10-\mathrm{C} 15-\mathrm{C} 14$ & $-179.8(3)$ \\
\hline $\mathrm{C} 3-\mathrm{C} 4-\mathrm{C} 5-\mathrm{C} 6$ & $0.9(4)$ & $\mathrm{C} 1-\mathrm{C} 9-\mathrm{N} 1-\mathrm{C} 10$ & $-179.8(2)$ \\
\hline $\mathrm{C} 3-\mathrm{C} 4-\mathrm{C} 5-\mathrm{Br} 1$ & $-178.1(2)$ & $\mathrm{C} 15-\mathrm{C} 10-\mathrm{N} 1-\mathrm{C} 9$ & $1.3(4)$ \\
\hline $\mathrm{C} 4-\mathrm{C} 5-\mathrm{C} 6-\mathrm{C} 1$ & $-0.7(4)$ & $\mathrm{C} 11-\mathrm{C} 10-\mathrm{N} 1-\mathrm{C} 9$ & $-178.3(2)$ \\
\hline $\mathrm{Br} 1-\mathrm{C} 5-\mathrm{C} 6-\mathrm{C} 1$ & $178.21(19)$ & $\mathrm{C} 4-\mathrm{C} 3-\mathrm{O} 2-\mathrm{C} 7$ & $-8.4(4)$ \\
\hline $\mathrm{C} 2-\mathrm{C} 1-\mathrm{C} 6-\mathrm{C} 5$ & $0.7(4)$ & $\mathrm{C} 2-\mathrm{C} 3-\mathrm{O} 2-\mathrm{C} 7$ & $171.4(2)$ \\
\hline $\mathrm{C} 9-\mathrm{C} 1-\mathrm{C} 6-\mathrm{C} 5$ & $-178.0(2)$ & $\mathrm{C} 8-\mathrm{C} 7-\mathrm{O} 2-\mathrm{C} 3$ & $-172.8(2)$ \\
\hline $\mathrm{C} 2-\mathrm{C} 1-\mathrm{C} 9-\mathrm{N} 1$ & $0.6(4)$ & $\mathrm{C} 12-\mathrm{C} 11-\mathrm{O} 3-\mathrm{C} 16$ & $-6.0(4)$ \\
\hline
\end{tabular}




$\begin{array}{lll}\mathrm{C} 6-\mathrm{C} 1-\mathrm{C} 9-\mathrm{N} 1 & 179.3(2) & \mathrm{C} 10-\mathrm{C} 11-\mathrm{O} 3-\mathrm{C} 16\end{array}$

Hydrogen-bond geometry $\left(A,{ }^{\circ}\right)$

$\mathrm{Cg} 1$ and $\mathrm{Cg} 2$ are the centroids of the $\mathrm{C} 1-\mathrm{C} 6$ and $\mathrm{C} 10-\mathrm{C} 15$ rings, respectively.

\begin{tabular}{lllll}
\hline$D-\mathrm{H} \cdots A$ & $D-\mathrm{H}$ & $\mathrm{H} \cdots A$ & $D \cdots A$ & $D-\mathrm{H} \cdots A$ \\
\hline $\mathrm{O} 1-\mathrm{H} 1 \cdots \mathrm{N} 1$ & $0.81(5)$ & $1.80(5)$ & $2.566(3)$ & $157(5)$ \\
$\mathrm{C} 16-\mathrm{H} 16 A \cdots \mathrm{O} 1^{\mathrm{i}}$ & 0.96 & 2.55 & $3.293(3)$ & 135 \\
$\mathrm{C} 7-\mathrm{H} 7 A \cdots C g 1^{\mathrm{ii}}$ & 0.97 & 2.80 & $3.662(3)$ & 149 \\
$\mathrm{C} 13-\mathrm{H} 13 \cdots C g 2^{\mathrm{iii}}$ & 0.93 & 2.79 & $3.629(3)$ & 150 \\
\hline
\end{tabular}

Symmetry codes: (i) $-x+1,-y,-z+1$; (ii) $-x+1 / 2, y+1 / 2,-z+1 / 2$; (iii) $-x+3 / 2, y-1 / 2,-z+1 / 2$. 